\section{TRANSTORNOS MENTAIS EM TRABALHADORAS DO ESTADO DA BAHIA}

\section{MENTAL DISORDERS IN FEMALE WORKERS OF BAHIA STATE}

Edsônia dos Santos Barbosa Ribeiro ${ }^{1} /$ Ludilvania Almeida Silva ${ }^{1}$ / Marcela Andrade Rios ${ }^{1 /}$ Rebeca de Jesus Silva ${ }^{1}$ / Tânia Teixeira de Figueredo ${ }^{1, *}$

\section{INTRODUÇÃO}

A saúde mental não apresenta uma única definição, uma vez que as diferenças culturais, religiosas, sociais interferem em seu conceito. Os níveis cognitivos ou emocionais interferem na condição de saúde do indivíduo, onde os transtornos mentais fazem parte das doenças crônicas não transmissíveis (DCNT), causando danos psicossociais (OMS, 2002).

Nos últimos anos, o quantitativo de indivíduos acometidos por comprometimentos de saúde mental apresenta crescimento, especialmente aqueles caracterizados como transtornos mentais comuns (TMC) que apresentam sintomas como: insônia, fadiga, irritabilidade, esquecimento, dificuldade de concentração e queixas somáticas (SILVA et al., 2018).

Indivíduos de países ocidentais apresentam prevalência de transtornos mentais não psicóticos que variam de $7 \%$ a $26 \%$, sendo mais elevadas no sexo feminino do que no masculino (ARAÚJO; PINHO; ALMEIDA, 2005).

Dentre os tipos de transtornos existentes, a depressão é um dos mais prevalentes e vem ocupando a terceira posição de problema de saúde entre mulheres moradoras de países desenvolvidos e a quinta nos países em desenvolvimento. São prevalentes não somente sintomas depressivos, mas transtornos relacionados aos fatores psicossociais e ambientais, como transtornos de ansiedade, transtornos de ajustamento, insônia, estresse, transtorno da alimentação e anorexia nervosa (ARAÚJO; PINHO; ALMEIDA, 2005).

Neste sentido multifatorial dos transtornos mentais, inserem-se as questões laborais das mulheres, pois muitas delas desenvolvem uma diversidade de atividades que propiciam o desencadeamento de ocorrência de agravos à saúde mental (BRASIL, 2003).

\section{RESUMO}

Objetivo: Descrever os casos de transtornos mentais envolvendo trabalhadoras do estado da Bahia. Materiais e métodos: Trata-se de um estudo descritivo, transversal acerca dos transtornos mentais em trabalhadoras da Bahia, entre os anos de 2009 e 2018, por meio de dados obtidos por acesso eletrônico a Diretoria de Vigilância e Atenção à Saúde do Trabalhador, com base em informações do Sistema de Informações de Agravos e Notificações (SINAN). Resultados: Foram notificados 334 casos de transtornos mentais em mulheres trabalhadoras, com maior prevalência no ano de 2018, na faixa etária de 30 a 39 anos, raça/cor ignorada/em branco. Mais da metade das trabalhadoras possuíam emprego registrado, com zona de residência predominante em Salvador, as atividades realizadas "ignoradas" foram mais frequentes. A principal evolução encontrada foi de incapacidade temporária. Conclusão: este estudo denota predominância nos transtornos depressivos, ansiedade e estressores que são transtornos comprometedores da vida laboral das mulheres.

Palavras-chave: Saúde do trabalhador. Saúde da mulher. Transtorno mental. Saúde mental.

\section{ABSTRACT}

Objective: To describe the cases of mental disorders involving workers from the state of Bahia. Materials and methods: This is a descriptive, cross-sectional study about mental disorders in workers from Bahia, between the years 2009 and 2018, through data obtained by electronic access to the Directorate of Surveillance and Attention to Worker's Health, based on information from the Diseases Information System and Notifications (SINAN). Results: 334 cases of mental disorders in working women were reported, with a higher prevalence in 2018, aged 30 to 39 years, race / color ignored / blank. More than half of the workers had registered employment, with a predominant area of residence in Salvador, the activities performed "ignored" were more frequent. The main evolution found was temporary disability. Conclusion: this study shows a predominance of depressive disorders, anxiety and stressors that are disorders that compromise women's working life.

Keywords: Worker's Health. Women's Health. Mental Disorder. Mental Health.

Submetido em: 08 de nov. 2019

Aceito em: 14 de fev. 2020

${ }^{1}$ Universidade do Estado da Bahia - UNEB, Guanambi, Bahia - Brasil.

E-mail para correspondência: figueiredotania70@gmail.com 
Os problemas mentais oriundos do processo de trabalho, muitas vezes, decorrem de crises geradas pelas características de monotonia, repetitividade, desvalorização e pelas demandas dos papéis sociais as quais a mulher deve atender (ARAÚJO; PINHO; ALMEIDA, 2005).

Este estudo tem como objetivo descrever os casos de transtornos mentais envolvendo trabalhadoras do estado da Bahia, entre os anos de 2009 a 2018.

\section{MATERIAL E MÉTODOS}

Trata-se de um estudo descritivo e transversal acerca dos transtornos mentais em trabalhadoras da Bahia, notificados entre os anos de 2009 e 2018, no Sistema de Informações de Agravos e Notificações (SINAN). Os dados foram obtidos por acesso eletrônico a Diretoria de Vigilância e Atenção à Saúde do Trabalhador (DIVAST), o qual reúne dados de eventos e agravos englobando trabalhadores da Bahia.

Foram selecionados os casos enquadrados como transtornos mentais e envolvendo o sexo feminino.

A análise das variáveis englobou os quesitos sociodemográficos e laborais (sexo, raça/cor, faixa etária, seção de atividade econômica, zona de residência e mercado de trabalho) e em relação aos transtornos mentais (ano de notificação, diagnóstico específico, evolução, emissão de comunicação de acidentes de trabalho - CAT).

Os dados foram tabulados e analisados com auxílio do Microsoft Office Excel, versão 2010, o qual possibilitou a realização do cálculo das frequências relativas.

Pelo fato dos dados serem provenientes de um banco de domínio público e sem identificação das traba- lhadoras, não houve submissão comitê de ética em pesquisa.

\section{RESULTADOS E DISCUSSÃO}

Um total de 334 casos de transtornos mentais envolvendo mulheres trabalhadoras do no período estudado. Os anos de maior incidência foram2018 $(\mathrm{n}=66 ; 19,8 \%)$, seguido por 2017 ( $n=48 ; 14,4 \%)$.

Ao analisar as características sociodemográficas e laborais das trabalhadoras, as faixas etárias com maiores quantitativos de notificação foram as de 30 a 39 anos $(n=135 ; 40,4 \%)$ seguida por de 40 a 49 anos $(n=114$; $34,1 \%$ ).

Tais faixas etárias abarcam a população economicamente ativa e infere-se que as trabalhadoras já teriam algum tempo de experiência laboral, podendo aumentar o tempo de exposição a riscos, tais como estresse laboral que contribuem para o desenvolvimento de transtornos mentais comuns (GUIMARÃES; SANTOS, 2019).

Quanto à raça/cor, a categoria referente a casos os ignorados/brancos apresentou números elevados $(n=139$; 41,6\%), demonstrando a existência de subregistros, o que dificulta uma visualização mais completa da problemática. A cor parda correspondeu a $34,4 \%$ dos casos. O estado da Bahia apresenta a raça negra (somando-se pretos e pardos) como predominante de forma autorreferida (IBGE, 2019).

A situação do mercado de trabalho entre esses casos corresponde uma maior frequência em pessoas que tem emprego registrado $(n=184 ; 55,1 \%)$. A trabalhadora com emprego formalizado tem maior acesso a serviços de saúde e, consequentemente, maiores registros de problemas de saúde, quando comparados aos trabalhadores informais (MIQUILIN et al., 2013).
Ressalta-se que foram encontradas notificações para indivíduos desempregados, demonstrando que os transtornos mentais relacionados ao trabalho são, muitas vezes, de natureza crônica e podem ter os seus sinais e sintomas iniciados mesmo quando a trabalhadora já está desligada do seu processo laboral.

Em relação à zona de residência, o município que mais notificou casos foi à capital baiana, Salvador, com $31,7 \%$, seguida por Feira de Santana, com $25,1 \%$.

Também foram investigados as atividades econômicas das trabalhadoras, as quais tem a maior prevalência em "ignorado" com 59,9\%, seguidamente de "Atividades profissionais, científicas e técnicas", "Atividades financeiras, de seguros e serviços" cada uma com respectivamente $11,7 \%$ e $9 \%$.

Em decorrência da dificuldade de encontrar empregos, muitos indivíduos são sujeitos a atividades precárias, baixa remuneração, insatisfações, excesso de carga de trabalho gerando sobrecarga mental e pouco domínio em suas atividades laborais. As cidades que apresentaram maior quantitativo populacional contribuem para os maiores índices (MASCARENHAS, 2014; IBGE, 2019).

Referente ao diagnóstico específico do transtorno (tabela 2), a maior frequência foi de "transtornos fóbicosansiosos com um percentual de 20,1\% em $n=67$ casos. Seguido de "estado de stress pós-traumático" com 36 casos, correspondendo a 10,8\%, e “episódios depressivos" $8,7 \%$ que equivale a 29 notificações. A ocorrência destes transtornos geralmente está ligada a condições de trabalhos que podem produzir riscos psicossociais, tais como: cargas de trabalho excessivas, comunicação ineficaz, falta de apoio e 
interação na relação chefe e empregador e colega com colega, insegurança laboral e condições precárias. Sendo um desafio e preocupação aos órgãos responsáveis a segurança e saúde do trabalhador (MINISTERIO DA SÁUDE, 2017).

Tabela 1 - Características sociodemográficas de investigação por transtorno mental em trabalhadoras na Bahia, no período de 2009 a 2018.

\begin{tabular}{|c|c|c|}
\hline VARIÁVEIS & $\mathbf{n}$ & $\%$ \\
\hline \multicolumn{3}{|l|}{ Faixa etária } \\
\hline Inconsistente* & 1 & 0,3 \\
\hline 20 a 29 anos & 34 & 10,2 \\
\hline 30 a 39 anos & 135 & 40,4 \\
\hline 40 a 49 anos & 114 & 34,1 \\
\hline 50 a 59 anos & 49 & 14,7 \\
\hline 60 a 69 anos & 1 & 0,3 \\
\hline \multicolumn{3}{|l|}{ Raça/cor } \\
\hline Ignorados/branco & 139 & 41,6 \\
\hline Branca & 51 & 15,3 \\
\hline Preta & 26 & 7,8 \\
\hline Parda & 116 & 34,7 \\
\hline Indígena & 2 & 0,6 \\
\hline \multicolumn{3}{|l|}{ Situação no mercado de trabalho } \\
\hline Ignorados/branco & 15 & 4,5 \\
\hline Empregado registrado & 184 & 55,1 \\
\hline Empregado não registrado & 2 & 0,6 \\
\hline Autônomo & 3 & 0,9 \\
\hline Servidor Público Estatutário & 55 & 16,5 \\
\hline Servidor Público Celetista & 24 & 7,2 \\
\hline Aposentado & 4 & 1,2 \\
\hline Desempregado & 26 & 7,8 \\
\hline Trabalho Temporário & 3 & 0,9 \\
\hline Cooperativado & 10 & 3 \\
\hline Empregador & 1 & 0,3 \\
\hline Outros & 7 & 2,1 \\
\hline Total & 334 & 100 \\
\hline
\end{tabular}

Fonte: SINAN Net-Sistema de Informações de Agravos de Notificação, 2019.

Na tabela 2 podem ainda ser visualizados os dados de "evolução" do período de 2009 a 2018. Em 72,5\% dos casos as trabalhadoras com transtornos mentais apresentaram a incapacidade temporária. A "cura" desses transtornos representou $1,2 \%$, seguida por "cura não confirmada" com 3,3\%. Trabalhadores com transtornos mentais apresentam incapacidade para o funcionamento de suas atividades plenamente, com grande número de dias improdutivos. E acredita-se que, uma das principais causa de desfalque no trabalho é depressão (GUIMARAES, 2006).
Tabela 2 - Variáveis sobre os transtornos mentais relacionados ao trabalho em mulheres baianas, no período de 2009 a 2018.

\begin{tabular}{|c|c|c|}
\hline Variáveis & $\mathbf{n}$ & $\%$ \\
\hline \multicolumn{3}{|l|}{ Diagnóstico Específico } \\
\hline não preenchida & 21 & 6,3 \\
\hline F03 Demência não especificada & 1 & 0,3 \\
\hline $\begin{array}{l}\text { F064 Transtornos da ansiedade orgâni- } \\
\text { cos }\end{array}$ & 1 & 0,3 \\
\hline $\begin{array}{l}\text { F07 Transtornos de personalidade e do } \\
\text { comportamento devidos a do }\end{array}$ & 1 & 0,3 \\
\hline F201 Esquizofrenia Hebefrênica & 1 & 0,3 \\
\hline F25 Transtornos Esquizoafetivos & 1 & 0,3 \\
\hline $\begin{array}{l}\text { F29 Psicose nao-orgânica não especifi- } \\
\text { cada }\end{array}$ & 3 & 0,9 \\
\hline F30 Episodio Maníaco & 1 & 0,3 \\
\hline F31 Transtorno afetivo bipolar & 9 & 2,7 \\
\hline F32 Episódios depressivos & 29 & 8,7 \\
\hline F341 Distimia & 2 & 0,6 \\
\hline $\begin{array}{l}\text { F380 Outros transtornos do humor } \\
\text { [afetivos] isolados }\end{array}$ & 1 & 0,3 \\
\hline F40 Transtornos fóbico-ansiosos & 67 & 20,1 \\
\hline F42 Transtorno obsessivo-compulsivo & 7 & 2,1 \\
\hline $\begin{array}{l}\text { F43 Reações ao stress grave e trans- } \\
\text { tornos de adaptação }\end{array}$ & 16 & 4,8 \\
\hline F430 Reação aguda ao stress & 16 & 4,8 \\
\hline F431 Estado de stress pós-traumático & 36 & 10,8 \\
\hline F432 Transtornos de adaptação & 10 & 3 \\
\hline $\begin{array}{l}\text { F439 Reação não especificada a um } \\
\text { stress grave }\end{array}$ & 2 & 0,6 \\
\hline $\begin{array}{l}\text { F488 Outros transtornos neuróticos } \\
\text { especificados }\end{array}$ & 9 & 2,7 \\
\hline $\begin{array}{l}\text { F60 Transtornos específicos da perso- } \\
\text { nalidade }\end{array}$ & 3 & 0,9 \\
\hline F920 Distúrbio depressivo de conduta & 1 & 0,3 \\
\hline F99 Transtorno mental & 17 & 5,1 \\
\hline $\begin{array}{l}\text { V730 Ocupante de um ônibus trauma- } \\
\text { tizado em colisão com um automóvel }\end{array}$ & 1 & 0,3 \\
\hline Z730 Esgotamento & 8 & 2,4 \\
\hline \multicolumn{3}{|l|}{ Evolução do caso } \\
\hline Cura & 4 & 1,2 \\
\hline Cura não confirmada & 11 & 3,3 \\
\hline Incapacidade Temporária & 242 & 72,5 \\
\hline Incapacidade Permanente Parcial & 27 & 8,1 \\
\hline Incapacidade Permanente Total & 2 & 0,6 \\
\hline Outra & 12 & 3,6 \\
\hline \multicolumn{3}{|l|}{ Emissão da CAT } \\
\hline Sim & 146 & 43,7 \\
\hline Não & 125 & 37,4 \\
\hline Ignorados/Branco & 42 & 12,6 \\
\hline
\end{tabular}

Fonte: SINAN Net-Sistema de Informações de Agravos de Notificação, 2019

Quanto à variável emissão da comunicação de acidente de trabalho (CAT) em $43,7 \%$ houve o registro deste importante documento. Ressaltase que somente para as trabalhadoras que possuem carteira assinada e regidas pela Consolidação das Leis Trabalhistas existe a obrigatoriedade de emissão de tal documento. Este dado pode ser justificado pelas vivências no âmbito de trabalho, em alguns casos, as relações funcionário e patrão são marcadas pelo autoritarismo, desvalorização de sua capacidade e exigências demasiadas.
Outro quesito é grande competitivamente que ocorre entre os colegas de trabalho em uma demanda constante por produtividade e superioridade, assim contribuindo para o adoecimento mental (MINISTERIO DA SAÚDE, 2017).

\section{CONCLUSÃO}

Diante da análise dos dados foi possível levantar suposições de uma relação difícil entre o trabalho e os transtornos meta, pois às vivencias no ambiente de trabalho tornam-se determinantes no estado de saúde.

Foram encontradas maiores frequências de transtornos do tipo fóbicos, de estresse pós-traumático e depressivos, em mulheres de 30 a 39 anos de idade, com empregos registrados.

As informações feitas neste estudo fortalecem a necessidade de pautar abordagens mais ampla na assistência à saúde das trabalhadoras, sendo capaz de analisar as percepções destas mulheres entre as condições objetivas e subjetivas no cotidiano da vida e do trabalho de mulheres.

\section{REFERÊNCIAS}

ARAÚJO T.M., PINHO, P.S., ALMEIDA M.G. Prevalência de transtornos mentais comuns em mulheres e sua relação com as características sociodemográficas e o trabalho doméstico. Revista Brasileira de Saúde Materno Infantil. n.5, v3, p. 337 348.2005.

BRASIL. Ministério da Saúde. Profissionalização de auxiliares de enfermagem: cadernos do aluno: saúde mental - 2. ed. Brasília: Ministério da Saúde; Rio de Janeiro: Fiocruz, 2003.

CARVALHO. J; AGUIAR, M. G. G. Qualidade de vida e condições de trabalho de feirantes. Revista de Saúde 
Coletiva da UEFS, v. 7, n. 3, p. 6065, 2017.

GUIMARÃES, L. A.M et al. Prevalência de transtornos mentais em trabalhadores de uma universidade pública do Estado de São Paulo. Revista Brasileira de Saúde Ocupacional, v. 31, n. 113, p. 7-18, 2006.

GUIMARÃES, T.A.A.; SANTOS, R.L.C. A remanicomialização do cuidado em saúde mental no Brasil no período de 2010-2019: análise de uma conjuntura antirreformista. O Social em Questão, Rio de Janeiro, n. 44, p. 111-138, 2019.

IBGE. Cidades e Estados. 2019. Disponível em:

https://www.ibge.gov.br/cidadeseestados/ba.html? Acessado em 10/10/2019.

IPEA, Instituto de Pesquisa Econômica Aplicada. Carta de conjuntura. Disponível em: http://www.ipea.gov.br/cartadeconjunt ura/index.php/category/mercado-detrabalho/ acessado 10/10/2019.

MINISTERIO DA SÁUDE, Transtornos mentais são a $3^{\mathrm{a}}$ principal causa de afastamentos de trabalho, disponívelem:http://www.blog.saude.gov.br/inde x.php?option $=$ com_content\&view $=$ arti cle \&id $=52979 \&$ catid $=579 \&$ Itemid $=50$ 218 acessado em: 11/10/2019.

\section{SECRETARIA DE SAÚDE DE CU-}

RITIBA, Definição de Saúde Mental.

Disponível em:

http://www.saude.pr.gov.br/modules/c onteudo/conteudo.php?conteudo $=1059$ acessado em: 08/10/19.

SILVA P.A.S., et al. Prevalência de transtornos mentais comuns e fatores associados entre idosos de um município do Brasil. Ciências e Saúde coletiva. n.23, v 2, p.639-646, 2018. 